MKG-Chirurg 2016 9 9:73-75

DOI 10.1007/s12285-016-0049-2

Online publiziert: 27. April 2016

(c) Springer-Verlag Berlin Heidelberg 2016

CrossMark

\author{
H. Terheyden ${ }^{1} \cdot$ M. Bonsmann ${ }^{2} \cdot$ H.-P. Ulrich ${ }^{3}$ \\ ${ }^{1}$ Mund-, Kiefer- und Gesichtschirurgie, Rotes Kreuz Krankenhaus Kassel Gemeinnützige GmbH, Kassel, \\ Deutschland \\ ${ }^{2}$ MKG-Praxis, Düsseldorf, Deutschland \\ ${ }^{3}$ MKG-Chirurgie LindenArcaden, Lübeck, Deutschland
}

\title{
Make it simple?
}

\section{Chirurgische Ursachen von Implantatverlust}

So einfach, wie es häufig dargestellt wird, ist die Implantologie nicht. Das wird beim Lesen dieses Heftes klar, in dem es um Ursachen des Implantatverlustes geht, die eben zum Teil auch iatrogen sind.

Statistisch gesehen sind Implantate immer noch die sicherste Form des Zahnersatzes, besser als Kronenbrückenprothetik und besser als abnehmbare prothetische Arbeiten auf natürlichen Zähnen. Aber $95 \%$ Erfolg in Studien bedeuten eben auch $5 \%$ Misserfolge und diese treffen den einzelnen Patienten zu $100 \%$, da hilft im Einzelfall die Statistik dem Arzt/der Ärztin und seinem enttäuschten Patienten wenig.

Verschärfend kommt hinzu, dass die Implantologie keine Kassenleistung ist und viele Kassenpatienten erhebliche Zuzahlungen leisten. Dadurch ist in der Implantologie die Erwartungshaltung an den Effekt des investierten Kapitals und den Erfolg der implantologischen Maßnahme häufig besonders hoch, zumal die Implantologie im Publikum häufig zu mechanistisch wie ein Handwerk am unbelebten Objekt missverstanden wird. Der Beitrag des Patienten und seiner Gesundheit zum Implantaterfolg kommt im Artikel von Lethaus und Zöller zum Ausdruck.

Zudem haben viele Kollegen für sich entdeckt, dass Implantate lukrativ sind, was zu entsprechenden Werbeaktivitäten und Erfolgsversprechen in der Öffentlichkeit führt, bis hin zu 20 JahresErfolgsgarantien. Dies kann man wohl nur als Mischkalkulation unter Einbeziehung einer Versicherung verstehen, denn fachlich gesehen gibt es keine $100 \%$ ige Erfolgsrate in einem biologischen (kon- taminierten) Umfeld. All dies trägt zu einer kritischen gesellschaftlichen und politischen Beobachtung der Therapieform Zahnimplantat bei.

Wenn in dieser Situation eine wirtschaftliche Hinwendung vieler zahnärztlicher Praktiker zum Selbersetzen der Zahnimplantate zu beobachten ist, dann ist das zwar verständlich, auch weil viele Patienten aus Gründen der Bequemlichkeit häufig eine Implantatversorgung aus einer Hand fordern und dabei den operativ chirurgischen Faktor bei der Implantattherapie unterschätzen. Die Industrie muss auf diesen weltweiten Marktbedarf eingehen, indem sie minimalinvasive Konzepte und Produkte fördert, Therapien in der Werbung simplifiziert darstellt oder beispielsweise suggeriert, eine DVT-gestützte Bohrschablone könne chirurgische Fähigkeiten ersetzen.

Aber nihil fit sine causa, wie im Beitrag Stiller und Wiltfang in diesem Heft steht. Zur Rettung eines Implantats bei Periimplantitis werden in diesem Artikel ganz neue chirurgische Therapiewege beschritten. Langfristige biologische (Periimplantitis) oder technische Komplikationen (z. B. Materialbrüche bei größenreduzierten Implantaten) werden derzeit in der öffentlichen Wahrnehmung noch selten mit dem initialen Setzen der Implantate und der Qualität der prothetischen Versorgung in Verbindung gebracht. Noch wird eher ein Frühverlust als ein Spätverlust dem Operateur angelastet und der Jahre später auftretende Spätverlust wird meistens einer inadäquaten Mundhygiene zugeschrieben. Aber stimmt das, wenn in einer Reihe von
Implantaten bei einem Patienten nur ein Einzelnes von Periimplantitis befallen ist?

\section{》) Konsequente Nachsorge und parodontale Supportivtherapie werden wichtiger}

Ein Problem der immer breiteren Anwendung der Methode der Zahnimplantation ist eine Dunkelziffer des Implantatverlusts, abseits der hohen Erfolgsraten unter Studienbedingungen. In Querschnittserhebungen wird die Periimplantitisrate teilweise auf über $40 \%$ geschätzt und auch die Frühverlustrate liegt mit teilweise $5 \%$ über den Erwartungen. Auch die Bedeutung einer konsequenten Nachsorge und parodontalen Supportivtherapie wird immer deutlicher. Die Bevölkerung weiß dies schon lange, denn Verluste sprechen sich etwa zwanzigmal so schnell herum wie Implantaterfolge. Man muss nur einmal im Verwandtschafts- und Freundeskreis nachfragen, um die Skepsis zu erleben. Übrigens gibt in diesen Fällen sehr häufig der zahnlose atrophierte Oberkiefer Anlass zu Kummer.

Ein Facharzt kann im Regelfall Knochen und Weichteil adäquat augmentieren und damit Voraussetzungen für eine dauerhafte Implantatgesundheit auch unter schwierigen Bedingungen schaffen. Die Rechtsprechung fordert im Streitfall einen Facharztstandard, mit anderen Worten einen fachärztlichen oder facharztäquivalenten Ausbildungsstand. Die Kunst eines Chirurgen oder einer Chirurgin ist schwer messbar, aber „make it simple“ reicht in der implan- 
tologischen Praxis nicht aus, um die Patienten zu überzeugen, sondern eher die Behandler.

Es geht darum, die Behandlungsmethode Zahnimplantat maßvoll und kompetent einzusetzen und ihr in Ausbildung und fachlichem Standard einen hohen Rang einzuräumen, auch in der Ausbildung der MKG-Chirurgen. Die Implantologie und die präprothetische Chirurgie sollten im Fach nicht stiefmütterlich behandelt werden. Ein Grund für die Auswahl des Themenschwerpunkts dieses Heftes des MKG-Chirurgen ist es, im eigenen Gebiet etwas Werbung für die fachliche und wissenschaftliche Beschäftigung mit der Implantologie zu machen. Die Schwierigkeiten liegen, eben typisch für die Zahnheilkunde, im Detail und im Kleinen. Marginale Knochendefekte auf dem obersten Millimeter, die Gestaltung des epithelialen Abschlusses und des Weichteilbetts, schon geringe Implantatpositionierungsfehler können über Erfolg und Misserfolg entscheiden - eine Aufgabe für den Facharzt.

Viel Erfolg für Ihre Praxis und gute Anregungen beim Lesen wünschen

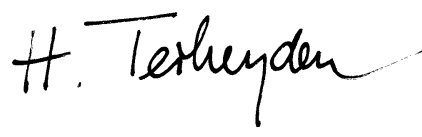

Prof. Dr. Dr. H. Terheyden

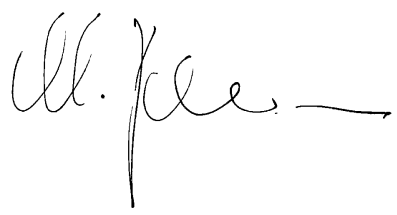

Dr. Dr. M. Bonsmann

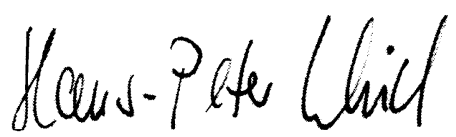

Dr. Dr. H.-P. Ulrich

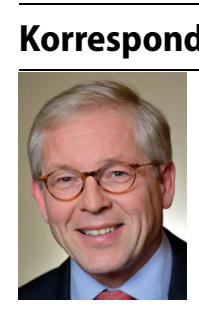

\section{Computergenerierte medizinische Diagnosen}

MRT, CT, Pathologie - Ärzte müssen immer mehr und immer komplexere medizinische Bilddaten berücksichtigen, um Diagnosen zu stellen und Therapien zu überwachen. Ein neuer Ansatz, an dem das FraunhoferInstitut für Bildgestützte Medizin MEVIS in Bremen gemeinsam mit der niederländischen Radboud-Universität Nijmegen arbeitet, soll sie dabei unterstützen. Beim Projekt AMI (Automation in Medical Imaging) sollen selbstlernende Computeralgorithmen die Datenfluten automatisch durchforsten und nach Auffälligkeiten suchen, um dadurch künftig die Treffsicherheit von computergenerierten Diagnosen zu steigern. Diese sogenannten Deep-Learning-Algorithmen können ihre Stärken vor allem dann ausspielen, wenn riesige $\mathrm{Da}$ tenmengen zu bewältigen sind, so MEVIS. Bei der Analyse geht es darum, feinste Unterschiede zwischen neueren und älteren

Interessenkonflikt. M. Bonsmann und H.-P. Ulrich geben an, dass kein Interessenkonflikt besteht. H. Terheyden gibt an, dass er in den vergangenen drei Jahren Forschungsmittel aus der ITI Stiftung und Honorare für Kurse und Kongressvorträge von den Firmen Straumann, Geistlich, Dentsply, Camlog erhalten hat. Tumoren im Frühstadium aufzuspüren. Ver-
Aufnahmen zu erkennen, um zum Beispiel änderungen von Form, Grauwert oder Textur können Rechner hervorragend unterscheiden und sogar selbst herausfinden, welche davon entscheidend sind.

Das AMI-Team will zunächst drei konkrete Beispiele entwickeln:

- Krebsfrüherkennung: Analyse der CT-Scans bei Risikopatienten, beispielsweise für Lungenkrebs.

- Augenheilkunde: Analyse von Laserscans von Patienten mit Erkrankungen der Netzhaut.

- Digitale Pathologie: Analyse von digitalisierten, hochaufgelösten Gewebeschnitten von Tumorpatienten und Aufspüren kleinster Mikrometastasen.

Quelle: Fraunhofer Institut, www.mevis.fraunhofer.de 
Hier steht eine Anzeige.

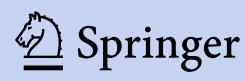

